Actualidades en Psicología, 32(124), 2018, 91-109

ISSN 2215-3535

http://revistas.ucr.ac.cr/index.php/actualidades

DOI: https://doi.org/10.15517/ap.v32i124.29021

\title{
Relação entre o pai e os filhos após o divórcio: revisão integrativa da literatura
}

\section{Relationship Between Father And Children After Divorce: Integrative Literature Review}

\author{
Joyce Lúcia Abreu Pereira Oliveira ${ }^{1}$ \\ Maria Aparecida Crepaldi ${ }^{2}$ \\ Universidade Federal de Santa Catarina, Brasil
}

\begin{abstract}
Resumo. A continuidade da relação entre o pai e os filhos após o divórcio favorece o desenvolvimento da criança e da família. Este estudo teve por objetivo apresentar uma revisão integrativa da literatura de pesquisas empíricas, acerca da relação entre o pai e os filhos após o divórcio/separação conjugal. Os resultados sugerem que: existem fatores que favorecem e fatores que dificultam a relação entre pai e filhos após o divórcio; essa relação exerce influências sobre o desenvolvimento de crianças e adolescentes; a paternidade de pais separados é marcada por especificidades; e, intervenções psicológicas com o pai divorciado são indicadas para melhorar o funcionamento de famílias binucleares. Destaca-se a relevância desta temática para a formação de profissionais das áreas da saúde, educação e jurídica, pois se trata de um fenômeno multifacetado, complexo e relevante para o desenvolvimento da criança e da família.
\end{abstract}

Palavras chave. Envolvimento paterno, pai, divórcio, separação conjugal, relações pai-criança.

Abstract. The continuity of the relationship between the father and the children after the divorce favors the development of the child and the family. This study aimed to present an integrative review of empirical research literature on the relationship between fathers and children after divorce/marital separation. The results suggests that: there are factors that favor and factors that hinder the relationship between fathers and children after divorce; this relationship exerts influence on the development of children and adolescents; separated-father parenting is marked by specific characteristics; and psychological interventions with divorced fathers are indicated to improve the functioning of binuclear families. This subject is highly relevant for the training of professionals in the health, education and legal fields, since it is a multifaceted phenomenon, complex and relevant for the development of children and family.

Keywords. Paternal involvement, father, divorce, marital separation, father-child relationships.

${ }^{1}$ Joyce Lúcia Abreu Pereira Oliveira.Universiade Federal de Santa Catarina, Brasil. Dirección Postal: Laboratório de Psicologia da Saúde, Família e Comunidade - Universidade Federal de Santa Catarina. Centro de Filosofia e Ciências Humanas. Departamento de Psicologia. Campus Reitor João David Ferreira Lima, s/n, Florianópolis-SC, Brazil. CEP 88.040-970. E-mail: joycelapo@gmail.com ${ }^{2}$ Maria Aparecida Crepaldi.Universiade Federal de Santa Catarina, Brasil. E-mail: maria.crepaldi@gmail.com

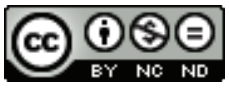

Esta obra está bajo una licencia de Creative Commons Reconocimiento-NoComercial-SinObraDerivada 4.0 Internacional. 


\section{Introdução}

A produção de pesquisas sobre a relação entre o pai e os filhos emergiu no contexto científico a partir das inúmeras transformações na ecologia da família, nos últimos 40 anos, ocasionadas por mudanças sociais, econômicas e culturais. Novas expectativas, crenças e atitudes sobre os papéis de pai e mãe têm sido gerados por fatores como o movimento feminista, a saída progressiva da mulher do ambiente doméstico e sua maior participação no mercado de trabalho, assim como o crescimento expressivo no número de separações e divórcios (Bossardi, Gomes, Vieira, \& Crepaldi, 2013; Cabrera, Tamis-LeMonda, Bradley, Hofferth, \& Lamb, 2000; Gomes, Bossardi, Cruz, Crepaldi, \& Vieira, 2014).

O divórcio é compreendido como uma transição de vida familiar, que acrescenta questões complexas para todos os membros da família ao exigir reorganizações estruturais, processuais e socioemocionais (Hetherington \& Kelly, 2002; Lamela \& Figueiredo, 2016). O estudo de Lamela, et al. (2010) evidenciou que a relação do pai com o filho, após a dissolução conjugal, interage de modo complexo com fatores individuais da mãe, do pai e do filho (em idade pré-escolar), além de fatores contextuais, sociais e culturais.

No contexto do divórcio/separação conjugal, pesquisadores têm destacado a importância da continuidade da relação entre o pai e a mãe e entre estes e os filhos após separação conjugal (Cezar-Ferreira \& Macedo, 2016; McGoldrick \& Shibusawa, 2016). Especialmente no que se refere à relação entre o pai e seus filhos, tendo em vista a constatação da importância do envolvimento e participação paterna para o desenvolvimento de crianças e adolescentes (Bandeira, 2013; Hack \& Ramires, 2010; Silva, 2012; Silva, 2003).

O estudo de Warshak (2014) evidenciou que a qualidade da relação entre o pai e a criança é favorecida quando os cuidados são compartilhados entre o pai e a mãe, e pernoites na casa do pai são um fator protetor, associado ao maior comprometimento do pai com o filho e menor incidência de afastamento paterno. Este estudo sugere que o envolvimento regular e frequente do pai com bebês e crianças menores de quatro anos deve ser incentivado.

A parentalidade do pai separado, no estudo realizado por Bandeira (2013) esteve muito influenciada pelo modelo tradicional, no qual o pai participa primordialmente através do provimento econômico, mas também pareceu influenciada pelos novos papéis sociais, no qual o pai ocupa-se com os cuidados básicos e estabelece envolvimento emocional com os filhos. A pesquisa de Silva (2003) encontrou um novo cenário no pós divórcio, caracterizado por pais mais envolvidos na criação dos ses filhos ou com o desejo de participar.

O envolvimento regular e frequente do pai com os filhos, após o término da conjugalidade, favorece o desenvolvimento da criança. E a privação paterna é considerada como um fator de risco, tanto em decorrência do divórcio, quanto pela baixa frequência de interações de pais que moram com os filhos (Cia, Williams, \& Aiello, 2005).

Nos estudos sobre paternidade, observa-se que há diferenças nas bases conceituais e nos níveis de análise sobre a temática. No que se refere aos termos mais utilizados nas publicações científicas sobre paternidade, destacam-se: envolvimento, engajamento e investimento parental. O conceito de engajamento paterno se refere a participação e preocupação contínua do pai biológico ou substituto em relação ao desenvolvimento e 
bem-estar físico e psicológico de seu filho (Dubeau, Devault, \& Paquette, 2009). E a concepção de investimento parental, se relaciona a perspectiva evolucionista e se refere ao cuidado psicológico e/ou biológico, que tem como propósito garantir a sobrevivência da prole e seu prosseguimento geracional, podendo ser direto (cuidados básicos) ou indireto (sustento financeiro da família) (Bossardi, 2011). Desse modo, observa-se que as bases conceituais diferem, mas parece haver o entendimento em comum de que existem especificidades na relação pai-criança, ou seja, a relação entre pai e filho difere da relação entre mãe e filho, produzindo influências/efeitos diferentes, sendo as duas relações importantes e fundamentais para o desenvolvimento infantil.

Diante da diversidade de elementos e aspectos que constituem as famílias, a utilização do pensamento sistêmico tem sido recomendada no desenvolvimento de pesquisas, sobretudo, a utilização dos pressupostos básicos da complexidade, instabilidade e intersubjetividade para compreensão dos fenômenos (Böing, Crepaldi, \& Moré, 2008). O pressuposto da complexidade contribui sobremaneira, devido a noção de contextualização, foco nas relações e na recursividade. Segundo Maturana (2014,p.74), recursão significa "a aplicação de uma operação sobre o resultado da aplicação de uma operação", assim, "cada vez que a repetição de um processo se aplica sobre as consequências de seu suceder anterior, se fala em recursão" (Maturana \& Yáñez, 2015, p. 543), o que inviabiliza explicações lineares e unicausais na análise dos fenômenos.

O pressuposto da instabilidade alude ao dinamismo das relações e a imprevisibilidade dos fenômenos, ou seja, considera que o mundo está em processo de tornar-se (Vasconcellos, 2010). Por fim, o pressuposto da intersubjetividade coloca a objetividade entre parênteses ao considerar a interdependência entre observador e objeto observado, ou seja, compreende a realidade como construção social, que emerge das distinções feitas pelo observador em espaços consensuais (Maturana, 2014a, 2014b; Maturana \& Varela, 2001).

$\mathrm{Na}$ atualidade, as pesquisas têm dado ênfase a famílias biparentais, o que se vê refletido na maior quantidade de publicações disponíveis na literatura (Vieira et al., 2014), mantendose uma lacuna sobre a paternidade em famílias nas diversas configurações familiares presentes na atualidade (Halme, Åstedt-Kurki, \& Tarkka, 2009). A revisão de literatura realizada por Gomes, Bossardi, Cruz, Crepaldi, \& Vieira (2014) evidencia a ausência considerável de estudos científicos com famílias separadas/divorciadas, recasadas e monoparentais. Nessa direção, pesquisadores têm evidenciado a relevância de novos estudos sobre o envolvimento paterno após o divórcio (Bossardi et al., 2013; Gomes et al., 2014; Halme et al., 2009; Lewis \& Dessen, 1999).

Quanto à relevância social deste estudo, destaca-se que conhecer os fatores que exercem influência sobre a relação pai-filho no contexto do divórcio, pode contribuir com a construção de políticas públicas que favoreçam a manutenção da relação entre pai e filhos, embasar a prática de profissionais que trabalham com famílias binucleares e auxiliar na construção de projetos de intervenção com famílias no pré e pós-divórcio.

Desse modo, considera-se que estudos sobre a temática são imprescindíveis para incrementar o conhecimento científico sobre o tema. Este estudo, portanto, buscou apresentar uma revisão integrativa da literatura de pesquisas empíricas, nacionais e internacionais, acerca da relação entre o pai e os filhos após o divórcio. 


\section{Método}

Realizou-se uma revisão integrativa da literatura, método indicado por Creswell (2007) para se organizar uma síntese de conhecimentos e proporcionar a incorporação da aplicabilidade dos resultados na prática. A execução desse estudo seguiu as cinco etapas sugeridas por Mendes, Silveira e Galvão (2008): (a) identificação do tema e seleção da hipótese ou questão de pesquisa para a elaboração da revisão integrativa; (b) estabelecimento de critérios para inclusão e exclusão de estudos/amostragem ou busca na literatura; (c) definição das informações a serem extraídas dos estudos selecionados/categorização dos estudos; (d) avaliação dos estudos incluídos na revisão integrativa; (e) apresentação da revisão/síntese do conhecimento.

A lista de descritores na Terminologia em Psicologia da BVS-Psi foi consultada e os termos considerados alinhados com o objetivo dessa pesquisa foram: divorce e father child relations, utilizados em inglês na busca realizada nas bases de dados internacionais: Pubmed, APA PsycNET e Web of Science. Utilizou-se o boleano AND para efetuar a busca de artigos. Os mesmos termos foram utilizados conjuntamente em língua portuguesa, em busca na base de dados Scielo Brasil, no entanto nenhum artigo foi localizado. Então, optou-se por fazer duas novas buscas nesta base de dados, uma utilizando apenas o termo divórcio e outra o termo relações pai-criança. A figura 1 apresenta o fluxograma do processo de seleção dos artigos empíricos.

A busca de artigos foi realizada no mês de março e atualizada em nove de dezembro de 2016. A partir da seleção dos artigos foram definidos como critérios de inclusão: estudos empíricos sobre a relação entre o pai e os filhos, após o divórcio/separação conjugal, publicados entre 2005 e 2016. A delimitação deste período de tempo ocorreu devido ao crescimento expressivo na taxa de divórcio nos últimos anos e o intuito de mapear e discutir a produção acadêmica recente. No Brasil houve um aumento de $160 \%$ nas taxas de divórcio na última década (IBGE, 2014), nos Estados Unidos entre 40 e 50\% dos casais se divorciaram (Greene, Anderson, Forgatch, DeGarmo, \& Hetherington, 2016; Owen \& Rhoades, 2012) e em Portugal as taxas também chegam a 50\% (Lamela et al., 2010).

Os artigos excluídos tratavam de temas relacionados a outros aspectos, tais como relação da criança com o padrasto, fatores de risco de incesto pai-filha, mortalidade e status marital, discursos jurídicos nas ações de divórcio, dentre outros. Desse modo, foram selecionados para análise 21 artigos internacionais e quatro estudos escritos em Português, totalizando 25 artigos. Destaca-se que um dos quatro artigos recuperados na base de dados Scielo Brasil trata-se de um estudo realizado em Portugal (Lamela, Castro, \& Figueiredo, 2010).

Os 25 artigos selecionados para análise foram lidos integralmente. Os dados para análise foram sistematizados em tabelas do software Excel e os resultados dos estudos foram analisados conforme análise categorial temática (Bardin, 2011).

\section{Resultados}

Primeiramente os resultados dos estudos incluídos nesta revisão foram agrupados nas categorias de análise: ano de publicação, tipo de análise de dados, participantes, tipo de estudo, sumarizados na tabela 1. Em seguida, apresenta-se a categoria principais resultados, 


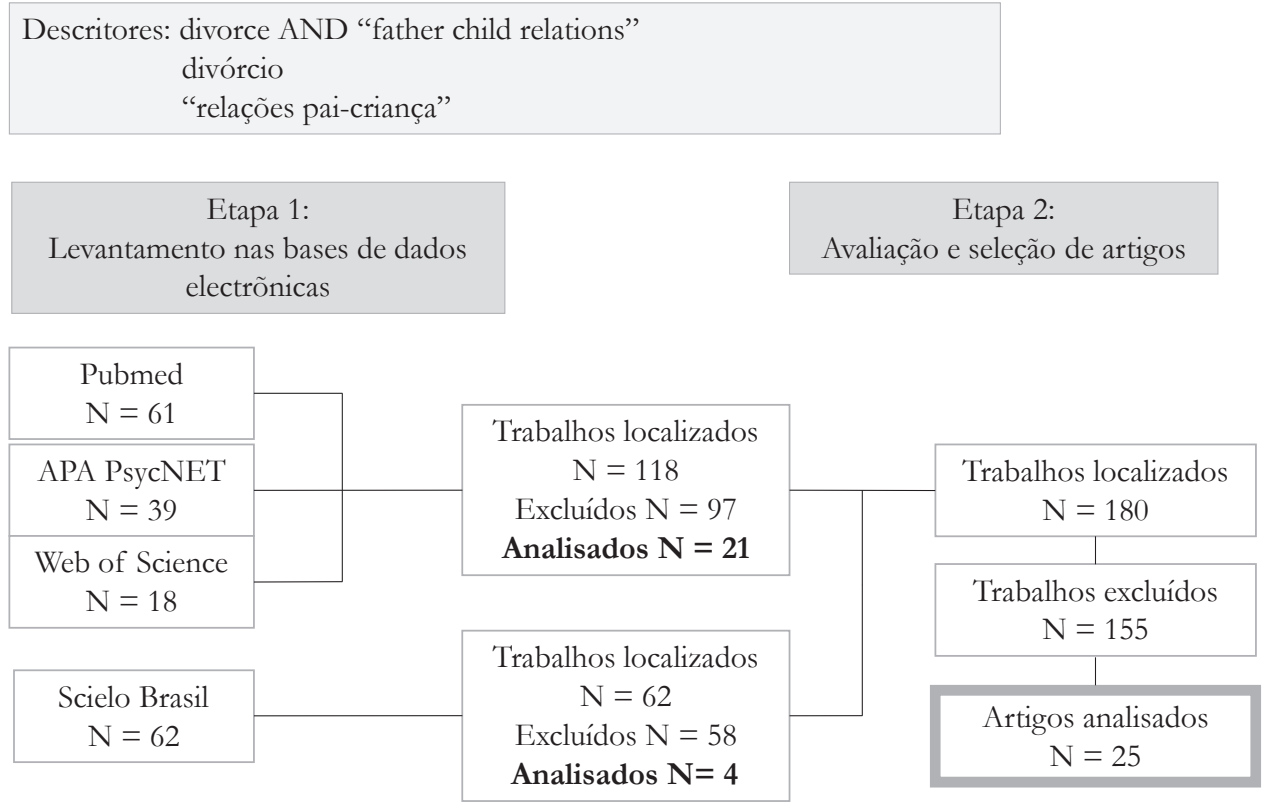

Figura 1. Fluxograma do proceso de seleção dos artigos empíricos.

sistematizada na tabela 2. Consistente com a maioria dos estudos empíricos, as pesquisas analisadas não fazem nenhuma distinção entre divórcio e separação conjugal (Yu, Pettit, Lansford, Dodge, \& Bates, 2010).

Dentre os resultados apresentados na tabela 1, destaca-se que o ano de 2010 teve o maior número de publicações, com cinco artigos. Quanto ao tipo de análise de dados, 22 trabalhos fizeram análises quantitativas e três análises qualitativas. Dentre as pesquisas qualitativas, duas são nacionais e uma internacional.

Com relação aos participantes, houve diversas variações, considerando a coleta de dados com o pai, a mãe e os filhos. Destaca-se que os filhos participaram de 20 estudos, sejam ele(a)s crianças, adolescentes, estudantes universitários ou jovens adultos (Kalmijn, 2015; Degarmo \& Forgatch, 2012; Klaus et al., 2012; Nobles, 2011; Yu, et al., 2010; Fabricius \& Luecken, 2007). A coleta foi realizada diretamente com o pai em nove estudos, dentre eles, Degarmo e Forgatch (2012), Lamela, Castro e Figueiredo (2010), Bastaits et al. (2012), Cookston et al. (2007), e de modo exclusivo na pesquisa de Cohen et al. (2014). A mãe participou de doze estudos, dentre eles, Elam et al., ( 2016); Nobles (2011); Kalil et al. (2011); Grzybowski \& Wagner (2010b); Grzybowski \& Wagner (2010a); Lamela, Castro \& Figueiredo (2010). 
Tabela 1

Sumário das característcas dos estudos

\begin{tabular}{|c|c|c|c|c|}
\hline $\begin{array}{l}\text { no. } \\
\text { artigo }\end{array}$ & $\begin{array}{l}\text { Estudo-Ano } \\
\text { Satisfacción }\end{array}$ & $\begin{array}{l}\text { Tipo de } \\
\text { estudo }\end{array}$ & $\begin{array}{l}\text { Tipo de } \\
\text { análise } \\
\text { de dados }\end{array}$ & Participantes \\
\hline 1 & Elam et al., ( 2016) & longitudinal & quanti & 240 mães e seus filho(a)s (9 e 12 anos) \\
\hline 2 & Kalmijn (2015) & transversal & quanti & 4.524 crianças \\
\hline 3 & $\begin{array}{l}\text { Cohen et al. } \\
\text { (2014) }\end{array}$ & transversal & quali & 20 pais \\
\hline 4 & $\begin{array}{l}\text { Degarmo \& } \\
\text { Forgatch (2012) }\end{array}$ & longitudinal & quanti & $\begin{array}{l}177 \text { pais (com e sem a custódia dos filhos) } \\
\text { e seu filho(a) ( } 4 \text { e } 11 \text { anos) }\end{array}$ \\
\hline 5 & $\begin{array}{l}\text { Bastaits et } \\
\text { al. (2012) }\end{array}$ & transversal & quanti & $\begin{array}{l}628 \text { filho(a)s ( } 10 \text { e } 18 \text { anos) e seus pais } \\
(224 \text { com pais casados e } 404 \text { com pais } \\
\text { divorciados) }\end{array}$ \\
\hline 6 & Klaus et al. (2012) & transversal & quanti & 739 adolescentes e jovens adultos \\
\hline 7 & Nobles (2011) & longitudinal & quanti & $\begin{array}{l}8.400 \text { famílias - adulto responsável pela } \\
\text { criança, em geral a mãe e a criança. }\end{array}$ \\
\hline 8 & Kalil et al. (2011) & longitudinal & quanti & $\begin{array}{l}\text { crianças, pai e mãe de } 15.992 \text { famílias } \\
\text { casadas com bebês nascidos entre os } \\
\text { anos } 1975-1979 \text {, cujos pais se divorciaram } \\
\text { durante a sua infância }\end{array}$ \\
\hline 9 & Yu, et al. (2010) & longitudinal & quanti & $\begin{array}{l}585 \text { participantes - mães com filhos de } 5 \text { a } \\
17 \text { anos e depois entrevista com o jovem } \\
\text { adulto aos } 22 \text { anos }\end{array}$ \\
\hline 10 & $\begin{array}{l}\text { Stamps et } \\
\text { al. (2009) }\end{array}$ & transversal & quanti & 4.663 adolescentes (11 e 19 anos) \\
\hline 11 & $\begin{array}{l}\text { Cookston et } \\
\text { al. (2007) }\end{array}$ & transversal & quanti & $\begin{array}{l}127 \text { pais, sendo que completaram o } \\
\text { programa } 98(77,2 \%) \text { e } 87 \text { pais no grupo } \\
\text { controle. As mães também participaram. }\end{array}$ \\
\hline 12 & $\begin{array}{l}\text { Fabricius \& } \\
\text { Luecken (2007) }\end{array}$ & transversal & quanti & 266 estudantes universitários \\
\hline 13 & Scott et al. (2007) & longitudinal & quanti & $\begin{array}{l}90.000 \text { estudantes do ensino médio e } \\
\text { fundamental }\end{array}$ \\
\hline 14 & $\begin{array}{l}\text { King \& } \\
\text { Sobolewski (2006) }\end{array}$ & transversal & quanti & 453 adolescentes \\
\hline 15 & $\begin{array}{l}\text { Finley \& } \\
\text { Schwartz (2006) }\end{array}$ & transversal & quanti & $\begin{array}{l}1.989 \text { estudantes universitários de famílias } \\
\text { biparentais e biucleares }\end{array}$ \\
\hline 16 & Flouri (2006) & transversal & quanti & 520 crianças em idade escolar \\
\hline
\end{tabular}

Continua... 


\begin{tabular}{|c|c|c|c|c|}
\hline $\begin{array}{l}\text { no. } \\
\text { artigo }\end{array}$ & $\begin{array}{l}\text { Estudo-Ano } \\
\text { Satisfacción }\end{array}$ & $\begin{array}{l}\text { Tipo de } \\
\text { estudo }\end{array}$ & $\begin{array}{l}\text { Tipo de } \\
\text { análise } \\
\text { de dados }\end{array}$ & Participantes \\
\hline 17 & $\begin{array}{l}\text { Hendricks et } \\
\text { al. (2005) }\end{array}$ & transversal & quanti & 1.409 adolescentes (11 e 18 anos) \\
\hline 18 & $\begin{array}{l}\text { Modecki et } \\
\text { al. (2015) }\end{array}$ & longitudinal & quanti & 156 adolescentes (15 a 19 anos) \\
\hline 19 & $\begin{array}{l}\text { Flouri, Narayanan } \\
\text { e Midouhas (2015) }\end{array}$ & longitudinal & quanti & $\begin{array}{l}\text { familias de pais residentes (15.293) e } \\
\text { famlias de pais não residentes (3.951) }\end{array}$ \\
\hline 20 & $\begin{array}{l}\text { Cheadle, Amato } \\
\text { \& King (2010) }\end{array}$ & longitudinal & quanti & 2.377 mães e 4.864 crianças \\
\hline 21 & $\begin{array}{l}\text { Mendle et } \\
\text { al (2009) }\end{array}$ & longitudinal & quanti & 1.382 filhos nascidos de 679 mães \\
\hline 22 & $\begin{array}{l}\text { Grzybowski \& } \\
\text { Wagner (2010a) }\end{array}$ & transversal & quali & 6 pais e 7 mães \\
\hline 23 & $\begin{array}{l}\text { Grzybowski \& } \\
\text { Wagner (2010b) }\end{array}$ & transversal & quanti & $\begin{array}{l}117 \text { pais e } 117 \text { mães separados / } \\
\text { divorciados com pelo menos um dos } \\
\text { filhos em idade escolar ( } 6 \text { a } 12 \text { anos) }\end{array}$ \\
\hline 24 & $\begin{array}{l}\text { Lamela, Castro \& } \\
\text { Figueiredo }(2010)\end{array}$ & transversal & quanti & 16 pais e mães \\
\hline 25 & Brito (2007) & transversal & quali & 30 adultos (21 a 29 anos) \\
\hline
\end{tabular}

Quanto a caracterização do tipo de estudo das pesquisas empíricas, identificou-se que 15 estudos foram transversais e 10 foram longitudinais. As coletas de dados dos estudos longitudinais tiveram diferentes etapas e espaços de tempo entre elas. Duas pesquisas longitudinais fizeram coleta de dados em dois momentos diferentes ao longo do tempo (Elam, Sandler, Wolchik, \& Tein, 2016; Nobles, 2011); outras duas empreenderam coleta de dados em três etapas (Degarmo \& Forgatch, 2012; Scott, Booth, King, \& Johnson, 2007); uma coletou em quatro etapas (Flouri, Narayanan, \& Midouhas, 2015) e cinco pesquisas acompanharam os participantes por um longo período de tempo, ou seja, da infância até a adolescência ou a fase jovem adulto (Modecki, Hagan, Sandler, \& Wolchik, 2015; Kalil, Mogstad, Rege, \& Votruba, 2011; Yu et al., 2010; Cheadle, Amato, \& King, 2010; Mendle et al., 2009).

\section{Principais resultados}

Os principais resultados dos estudos empíricos analisados foram organizados em seis subcategorias temáticas, que emergiram a partir da similaridade entre os resultados apresentados nos estudos analisados, quais sejam: Fatores que favorecem a relação entre o pai separado e seus filhos; Fatores que dificultam a relação entre o pai separado e seus filhos; Influências para o(a)s filho(a)s; Paternidade de pais separados; Intervenção psicológica com pais separados. 
Fatores que favorecem a relação entre o pai separado e seus filhos. Essa subcategoria incluiu condições destacadas na literatura, que favoreceram a relação entre o pai e seus filhos após separação conjugal. Morar com o pai e a mãe, após o término da conjugalidade, tem sido associado a relações mais favoráveis entre pai e filhos (Kalmijn, 2015; Scott et al., 2007). A coabitação foi evidenciada como um fator que interfere significativamente no envolvimento parental após a separação conjugal (Brito, 2007; Grzybowski \& Wagner, 2010b; Klaus, Nauk, \& Steinbach, 2012). Quanto mais tempo as crianças viveram com o pai após a separação/divórcio, melhores eram suas relações com ele (Cookston et al., 2007).

Evidências empíricas mostraram que o pai se envolve mais com os filhos quando a mãe trabalha fora de casa (Kalmijn, 2015). O pai com maior nível educacional relatou mais contato com os filhos, medido em termos de pernoites que o filho passou na casa do pai (Kalil et al., 2011; Kalmijn, 2015). A experiência familiar pré-divórcio (qualidade do relacionamento mãe-criança) e um atributo individual (sentimento de bem estar dos filho(a)s) mostraram ter grande influência na prevenção da deterioração do relacionamento entre o pai e os filhos (Scott et al., 2007).

Fatores que dificultam a relação entre o pai separado e seus filhos. Essa subcategoria incluiu condições, apresentadas na literatura, que dificultaram o envolvimento do pai com os filhos após o término da conjugalidade. O conflito entre os ex-cônjuges foi o fator mais destacado pelas pesquisas analisadas (Fabricius \& Luecken, 2007; Scott et al., 2007; Yu et al., 2010). Quanto mais os filho(a)s experimentaram o conflito entre o pai e a mãe, piores foram seus relacionamentos com seus pais e mais sofrimento sentiam, quando jovens adultos, sobre o divórcio dos pais (Fabricius \& Luecken, 2007). No entanto, o estudo longitudinal de Modecki, Hagan, Sandler e Wolchik (2015), evidenciou que mesmo quando a relação entre os ex-cônjuges era permeada por conflito elevado, o pai era altamente envolvido com os filhos.

A questão financeira foi apontada como um fator que gera muitos conflitos após a dissolução da conjugalidade (Grzybowski \& Wagner, 2010a) e o pai com maior nível educacional relatou maior conflito pós-divórcio com suas ex-esposas (Kalil et al., 2011). Os filhos serem colocados no centro das desavenças (Brito, 2007), o fato de o pai morar em outra casa e ser, geralmente, menos próximo dos filho(a)s antes da separação (Scott et al., 2007), foram evidenciados como aspectos que dificultaram a relação pai-filho(a)s após a separação/divórcio.

Influências para os filhos. Essa subcategoria incluiu as influências do envolvimento paterno para o desenvolvimento dos filhos, após a separação/divórcio, destacados nos estudos analisados. Foi evidenciado que o envolvimento regular e frequente do pai com os filhos, após o término da conjugalidade, favorece o desenvolvimento da criança (Degarmo \& Forgatch, 2012), sendo associado com níveis mais elevados de bem-estar para filhos e filhas (Stamps, Booth, \& King, 2009). A capacidade dos pais para estabelecer relações harmoniosas e boa coparentalidade, após o divórcio, mostrou-se um importante preditor de longo prazo do bem-estar de seus filho(a)s (Cookston et al., 2007). 
Nesse sentido, o envolvimento com o pai foi associado a menores problemas de internalização para filhas (Stamps et al., 2009) e a menores problemas de internalização e externalização, para adolescentes (King \& Sobolewski, 2006). Um outro estudo, longitudinal, encontrou crianças com maiores problemas de internalização e externalização, dois anos após o divórcio, quando o pai estava envolvido em alto nível de conflito com a ex-cônjuge e também seis anos mais tarde quando o pai tinha pouco contato com seus filhos e fornecia baixo apoio social (Elam et al., 2016). O estudo longitudinal realizado por Flouri, Narayanan e Midouhas (2015) mostrou que a ausência do pai na primeira infância, em famílias de pai não residente, aumenta o risco de comportamentos problemáticos (especialmente externalizantes) e afeta igualmente meninos e meninas.

Os estudos também evidenciaram que o pai tem a capacidade de aumentar a autoestima dos filhos (Bastaits, Ponnet, \& Mortelmans, 2012) e quanto mais convívio as crianças tiveram como o pai após a separação, melhores foram suas relações com eles quando jovens adultos (Fabricius \& Luecken, 2007). Destacou-se ainda, evidências de que a ausência do pai tem um efeito potencialmente negativo sobre as escolhas de vida dos adolescentes, relacionadas especificamente à atividade sexual (Hendricks et al., 2005) e que relações pobres entre pai-filho(a) e angústia sobre a separação dos pais, previu estado de saúde mais pobre em estudantes universitários (Fabricius \& Luecken, 2007). A ausência paterna também foi associada com atividade sexual precoce dos filhos (Mendle et al., 2009)

Paternidade de pais separados/divorciados. Essa subcategoria englobou particularidades da paternidade de pais separados/divorciados. Pais casados e divorciados, de diferentes grupos étnicos, apresentaram maior envolvimento instrumental (fornecimento de renda, proteção, desenvolvimento moral, disciplina) do que envolvimento expressivo (prestação de cuidados, companheirismo, compartilhamento de atividades, desenvolvimento emocional, social e espiritual) com os filhos (Finley \& Schwartz, 2006). O estudo de Grzybowski e Wagner (2010b) evidenciou que o envolvimento social (levar ao cinema e parque) de pais separados foi maior do que o de mães e que a experiência educativa, na perspectiva dos pais, foi marcada por perdas após a separação conjugal.

Quanto à qualidade do vínculo entre pai e filhos, um estudo revelou que essa relação não é afetada negativamente quando a mãe tem um novo parceiro (Kalmijn, 2015). No entanto, outra pesquisa mostrou que a proximidade emocional do filho(a) com a mãe, a duração da relação com o padrasto, e o casamento entre a mãe e o padrasto foram altamente preditivos para manter as relações da criança com o pai e o padrasto (Klaus et al., 2012). Outro estudo mostrou que a ligação entre o pai e seus filhos antes e após a separação conjugal influenciou na coparentalidade (Grzybowski \& Wagner, 2010a).

Um estudo longitudinal sobre os padrões de contato de pais separados, cujos filhos não coabitam com eles, mostrou que há diferentes trajetórias de contato do pai com os filhos e que a estabilidade de contato ao longo do tempo é mais comum do que a mudança (Cheadle, Amato, \& King, 2010). Os pesquisadores citados encontraram em um maior número de pais, um nível consistentemente alto de contato com seus filhos, cujas características incluíram, filhos mais velhos no momento da separação, mães mais 
velhas e com nível educacional mais alto e maior probabilidade do pai ser pagante de pensão alimentícia. O estudo longitudinal de Degarmo e Forgatch (2012) revelou que o fato do pai ter uma pessoa com quem possa compartilhar, em sua rede de apoio, previu para o pai melhores resultados na resolução de problemas, e que a resolução de problemas previu parentalidade mais eficaz, e esta, por sua vez, previu redução de problemas de comportamento nas crianças, ao longo de 18 meses.

O estudo de Nobles (2011) realizado no México, revelou que crianças de pais emigrantes têm mais contato com o pai, quando se compara a relação de pais divorciados com seus filhos. Os estudos também mostraram que os pais não-residentes devem ser encorajados a serem igualmente envolvidos com seus filhos e filhas (Stamps et al., 2009), tendo em vista que filhas têm maior probabilidade de terem relações negligenciadas pelos pais do que os filhos (Klaus et al., 2012) e os filhos relatarem que se sentem mais próximos de seus pais em comparação as filhas (Stamps et al., 2009).

A experiência de paternidade monoparental aparece em apenas um dos estudos analisados, o qual destacou que a guarda paterna exclusiva foi determinada pelo juiz e ocasionada pela incapacidade da mãe para ficar com o(a)s filho(a)s, devido a problemas psiquiátricos e/ou envolvimento com drogas (Cohen, Finzi-Dottan, \& Tangir-Dotan, 2014). A sobrecarga com a função parental, principalmente relacionada à guarda exclusiva foi mencionada tanto por pais (Cohen et al., 2014) quanto por mães (Grzybowski \& Wagner, 2010a).

Intervenção psicológica com pais separados/divorciados. Nessa subcategoria foram incluídas duas pesquisas sobre intervenções psicológicas realizadas com pais separados/divorciados. O programa Pais para a vida (Dads For Life) refere-se a uma intervenção em grupo com o pai separado sem a guarda dos filhos (Cookston et al., 2007). A intervenção também teve impacto nas mães, que não participaram da intervenção, pois tanto as mães quanto os pais relataram menos conflitos quando o pai participou da intervenção, em comparação com o grupo controle. Os pesquisadores evidenciaram que a intervenção oferece um programa promissor para melhorar o funcionamento das famílias após o divórcio.

O outro programa, Pais por Inteiro (PApI), refere-se a uma intervenção em grupo com pais e mães separados, distribuídos em dois grupos mistos, um experimental e um grupo controle (Lamela, Castro, et al., 2010). Como resultados os pesquisadores destacaram a melhora na adaptação ao divórcio, na coparentalidade e na binuclearidade familiar.

A tabela 2 buscou apresentar de modo integrado os resultados de estudos científicos que se encontram esparsos na literatura nacional e internacional.

\section{Discussão}

Quanto à caracterização dos estudos analisados, observou-se que houve um incremento no número de publicações após o ano 2010, o que parece demonstrar um interesse crescente nos estudos sobre a relação pai e filhos após a divórcio/separação conjugal. No que se refere ao tipo de estudo, a maioria foi do tipo transversal. A análise quantitativa foi o 
Tabela 2

Principais resultados

Categoria temática

Fatores que

favorecem a relação

entre o pai separado

e seus filhos

\section{Resultados}

Morar com o pai e a mãe (FatheKalmijn, 2015; Grzybowski \& Wagner, 2010b; Scott et al., 2007; Brito, 2007)

A mãe trabalhar fora de casa (FatheKalmijn, 2015)

Pai com maior nível educacional (Kalil et al., 2011; FatheKalmijn, 2015)

Padrões residenciais (Klaus et al., 2012)

O tempo em que as crianças viveram com o pai (Cookston et al., 2007)

A experiencia familiar pré-divórcio (Scott et al., 2007)

Fatores que dificultam a relação entre o pai separado e seus filhos

Conflito entre os ex-cônjuges (Kalil et al., 2011; Yu et al., 2010; Scott et al., 2007)

Pouca proximidade do pai com os filhos antes da separação (Scott et al., 2007)

O pai morar em outra casa (Scott et al., 2007)

Os filhos serem colocados no centro das desavenças (Brito, 2007)

A experiência de conflito entre o pai e a mãe (Fabricius \& Luecken, 2007)

Conflito entre o pai e a mãe e baixo contato e apoio do pai foi relacionado a maiores problemas de internalização e de externalização (Elam et al., 2016)

A ausência do pai foi relacionada a problemas de comportamento da criança (Flouri et al, 2015)

Envolvimento regular e frequente com o pai favorece o desenvolvimento da criança (Degarmo \& Forgatch, 2012)

Aumenta a auto-estima das crianças (Bastaits et al. 2012)

Redução de problemas de comportamento (Degarmo \& Forgatch, 2012)

A ausência paterna foi associada com atividade sexual precoce dos filhos (Mendle et al, 2009)

Menores problemas de internalização - para filhas (Stamps et al., 2009) e também de externalização - para adolescentes (King \& Sobolewski, 2006)

Níveis mais elevados de bem-estar para filhos e filhas (Stamps et al., 2009; Cookston et al., 2007)

Quanto mais convívio, melhores foram as relações como pai quando jovens adultos (Fabricius \& Luecken, 2007)

Relações pobres entre pai-filho(a) e angústia previu estado de saúde mais pobre (Fabricius \& Luecken, 2007)

A ausência do pai tem um efeito potencialmente negativo sobre escolhas de vida dos adolescentes (Hendricks, 2005)

Actualidades en Psicología, 32(124), 2018, 91-109

Continua... 
Tabela 2

Principais resultados

\begin{tabular}{|c|c|}
\hline Categoria temática & Resultados \\
\hline \multirow[t]{16}{*}{$\begin{array}{l}\text { Paternidade de pais } \\
\text { separados/divorciados }\end{array}$} & $\begin{array}{l}\text { A qualidade do vínculo com o pai não é afetada negativamente se a } \\
\text { mãe tem um novo parceiro (FatheKalmijn, 2015) }\end{array}$ \\
\hline & $\begin{array}{l}\text { Pais altamente envolvidos mesmo quando a relação com a ex-cônjuge } \\
\text { era permeada por conflitos (Modecki, et al, 2015) }\end{array}$ \\
\hline & $\begin{array}{l}\text { Sobrecarga com a função parental, relacionada a guarda exclusiva de } \\
\text { pais (Cohen et al., 2014) e de mães (Grzybowski, 2010). }\end{array}$ \\
\hline & $\begin{array}{l}\text { Paternidade monoparental ocasionada pela incapacidade da mãe } \\
\text { (Cohen et al., 2014) }\end{array}$ \\
\hline & $\begin{array}{l}\text { A proximidade emocional com a mãe, a duração da relação com o } \\
\text { padrasto, e o casamento entre a mãe e o padrasto são altamente preditivos } \\
\text { para as relações da criança com o pai e o padrasto (Klaus et al., 2012) }\end{array}$ \\
\hline & $\begin{array}{l}\text { Filhas têm maior probabilidade de ter relações negligenciadas com pais } \\
\text { do que os filhos (Klaus et al., 2012) }\end{array}$ \\
\hline & $\begin{array}{l}\text { Pais divorciados são susceptíveis de se beneficiar de programas de } \\
\text { formação de pais (Degarmo \& Forgatch, 2012) }\end{array}$ \\
\hline & $\begin{array}{l}\text { Crianças de pais emigrantes tem mais contato com o pai comparado } \\
\text { com a separação pai-filho de pais divorciados (Nobles, 2011) }\end{array}$ \\
\hline & $\begin{array}{l}\text { Coparentalidade relacionada a relação pai-filho(a)s antes do término da } \\
\text { união conjugal (Grzybowski \& Wagner, 2010a) }\end{array}$ \\
\hline & $\begin{array}{l}\text { Há repercussões da relação conjugal sob a parental (Grzybowski \& } \\
\text { Wagner, 2010b) }\end{array}$ \\
\hline & $\begin{array}{l}\text { O envolvimento social de pais apresentou-se maior que o das mães } \\
\text { (Grzybowski \& Wagner, 2010b) }\end{array}$ \\
\hline & $\begin{array}{l}\text { A experiência educativa do pai é marcada por perdas (Grzybowski \& } \\
\text { Wagner, 2010b) }\end{array}$ \\
\hline & $\begin{array}{l}\text { Contato mais frequente do pai quando os filhos são mais velhos no } \\
\text { momento da separação (Cheadle, Amato \& King, 2010) }\end{array}$ \\
\hline & $\begin{array}{l}\text { Filhos relataram que se sentem mais próximos de seus pais em } \\
\text { comparação com filhas (Stamps et al., 2009) }\end{array}$ \\
\hline & $\begin{array}{l}\text { Os pais devem ser encorajados a ser igualmente envolvidos com seus } \\
\text { filhos e filhas (Stamps et al., 2009) }\end{array}$ \\
\hline & Maior envolvimento instrumental que expressivo (Finley \& Schwartz, 2006) \\
\hline \multirow{2}{*}{$\begin{array}{l}\text { Intervenção } \\
\text { psicológica com pais } \\
\text { separados/divorciados }\end{array}$} & $\begin{array}{l}\text { Pais por Inteiro (PApI) - melhorou a adaptação ao divórcio, a coparentalidade } \\
\text { e a binuclearidade familiar (Lamela, Castro \& Figueiredo, 2010) }\end{array}$ \\
\hline & $\begin{array}{l}\text { Pais para a Vida (Dads For Life) - a intervenção teve impacto nas mães } \\
\text { (que não participaram da pesquisa) (Cookston et al., 2007) }\end{array}$ \\
\hline
\end{tabular}


tipo de análise de dados mais utilizada, tendo sido realizada em 22 estudos. Quanto aos participantes, os filhos foram incluídos na maioria das pesquisas, fato que parece demostrar o interesse de pesquisadores por variáveis que envolvam a saúde emocional e física dos filhos e a relação com o pai após a dissolução conjugal.

Dentre os principais resultados dos estudos levantados nesta revisão, o conjunto de autores que investigaram os fatores que favorecem o envolvimento do pai após o término da conjugalidade mostraram que a qualidade do relacionamento entre o pai e a mãe, exerce influências diretas sobre a relação entre o pai e os filhos, em consonância com os outros estudos com pais e/ou mães separadas (Bandeira, 2013; Hack \& Ramires, 2010; Silva, 2012; Silva, 2003). O envolvimento conjunto do pai e da mãe, nos cuidados e educação dos filhos, associou-se a relações mais próximas entre o pai e os filhos (King \& Sobolewski, 2006). A coabitação com o pai foi evidenciada como um importante fator protetor da relação entre o pai e os filhos, confirmando achados de Warshak (2014) que relacionam o maior comprometimento paterno com o fato de os filhos dormirem alguns dias da semana em sua casa.

O conflito interparental foi destacado como o fator que mais dificulta o envolvimento e participação do pai com os filhos após o término da conjugalidade. De um modo geral, quanto maior o nível de conflito entre os ex-cônjuges, mais baixos foram os níveis de contato e apoio do pai com os filhos, dados consoantes à pesquisa de Owen e Rhoades (2012).

Com relação às influências do pai para o desenvolvimento de crianças e adolescentes os estudos evidenciaram: maiores níveis de bem estar; menos sintomas emocionais; maior autoestima; melhor estado de saúde; e menores problemas de internalização e externalização, quando o pai separado manteve uma relação de proximidade física e emocional com os filhos, especialmente facilitada pela boa relação coparental. Tais achados corroboram o estudo realizado por Lamela e Figueiredo (2016) que investigou o impacto da coparentalidade no funcionamento psicológico dos filhos. Outro estudo demonstrou que a frequência de contato com o pai foi negativamente associada com sintomas emocionais da criança (Flouri, 2006).

Quanto à parentalidade do pai separado, alguns estudos mostraram pais mais envolvidos em atividades sociais e menos envolvidos em atividades de cuidado diário (dar banho, fazer comida, alimentar, vestir, colocar a criança para dormir) do que as mães, e outros anunciaram pais altamente envolvidos ou com o desejo de participar mais da vida dos filhos (Bandeira, 2013; Grzybowski, 2007; Silva, 2003). A parentalidade eficaz após a separação conjugal, com apoio e controle, é indicada como um fator protetor para o desenvolvimento dos filhos (Bastaits \& Mortelmans, 2016). Desse modo, o envolvimento regular e frequente do pai com os filhos, após o término da conjugalidade, favorece o desenvolvimento da criança. Assim, o novo pai caracterizado por Lamb $(1992,1997)$ ganha visibilidade em estudos científicos sobre a paternidade após o divórcio.

No que se refere à intervenções com pais separados foram destacadas: melhoras na adaptação ao divórcio, nos cuidados compartilhados e diminuição de conflitos entre os ex-cônjuges, fatores que interferem diretamente na relação pai-filho. Nesse contexto, o estudo de Zordan, Wagner e Mosmann (2012) afirmou que intervenções de profissionais 
da psicologia e da área jurídica devem considerar a complexidade na qual as relações familiares após o divórcio estão inseridas. Portanto, é importante evitar intervenções descontextualizadas ou normatizadoras, desconectadas da realidade social, econômica e cultural na qual cada família está imbricada.

Diante do exposto, os resultados desse estudo possibilitaram evidenciar a importância da continuidade da relação entre pai e filhos após o divórcio, especialmente devido os inúmeros benefícios para o desenvolvimento de crianças e adolescentes, consoante a outros estudos sobre a temática (Cezar-Ferreira \& Macedo, 2016; McGoldrick \& Shibusawa, 2016). A análise desse fenômeno deve observar a complexidade das relações familiares no contexto do término da conjugalidade (Lamela \& Figueiredo, 2016), ou seja, precisa considerar o contexto, o dinamismo e a recursividade das relações familiares (Böing et al., 2008).

\section{Considerações Finais}

Considerando os resultados desse estudo e a perspectiva sistêmica, para compreensão da relação entre pai e filhos após o divórcio, evidenciou-se a teia de relações na qual este fenômeno está imbricado, ou seja, há aspectos pessoais, relacionais e contextuais que interatuam um sobre o outro recursivamente, o que inviabiliza explicações lineares e unicausais. Desse modo, compreender as relações entre pai e filhos implica em: (a) considerar o contexto e o ciclo autoconstitutivo, autoorganizador e autoprodutor das relações familiares; (b) reconhecer a instabilidade e a imprevisibilidade, tanto no que se refere ao fato de que as pesquisas científicas apresentam evidências e não determinações sobre o fenômeno, quanto entender que as relações estão em constante processo de transformação ao longo do tempo; (c) assumir que a intersubjetividade do pesquisador e dos pesquisados estão contidas nos estudos científicos e são validados em espaços consensuais.

Diante disso, a organização da tabela 2 buscou mostrar uma síntese dos estudos sobre a temática, com o intuito de dar visibilidade aos principais aspectos apontados na literatura sobre a relação entre pai e filhos após o divórcio, e assim, facilitar a compreensão ampliada desse fenômeno através da análise de um conjunto de pesquisas. O ponto de vista da complexidade anuncia a necessidade dessa ampliação do foco de análise.

Para futuras pesquisas, sugere-se a consideração de fatores individuais da mãe, do pai e do filho, além de fatores contextuais, sociais e culturais. Estudos longitudinais com crianças e famílias binucleares poderiam investigar os fatores que promovem o desenvolvimento e relações saudáveis com o pai. No contexto brasileiro, sugere-se novos estudos sobre a relação entre o pai e os filhos após o divórcio, devido a lacuna apresentada na literatura, assim como estudos sobre programas de intervenção psicológica com pais e/ou mães separados, tendo em vista os efeitos benéficos das intervenções apresentadas neste estudo.

Presume-se que intervenções no pré e pós-divórcio poderiam beneficiar todos os membros da família. Especialmente, intervenções que privilegiassem a prevenção dos fatores de risco para o desenvolvimento dos filhos e da família, como rompimentos de relações entre pai, mãe, filhos, avós maternos e paternos.

Actualidades en Psicología, 32(124), 2018, 91-109 
Dentre as limitações desta pesquisa, estão o fato de existirem outras bases de dados que poderiam ter sido consultadas, o que aumentaria o número de pesquisas analisadas. Nova busca de artigos com outros descritores também poderia ampliar o número de estudos. Constatou-se a reduzida produção científica brasileira sobre relações entre o pai e os filhos após o divórcio. Desse modo, a necessidade de estudos nacionais sobre a temática parece evidente.

Diante deste cenário, destaca-se que conhecer as especificidades da relação do pai com os filhos após a separação/divórcio, pode propiciar orientação para a prática e intervenção de psicólogo(a)s, educadores, profissionais da área jurídica e da saúde que trabalham com famílias no pré e pós-divórcio, colaborando com a divulgação da importância do pai para o desenvolvimento da criança, do adolescente e da família. Assim, considera-se que estudos sobre a temática tem se mostrado atuais, especialmente os que embasam o exercício profissional.

\section{Referências}

Bandeira, R. K. B. (2013). A paternidade após o rompimento conjugal. Dissertação. Dissertação (Mestrado em Psicologia). Programa de Pós-Graduação em Psicologia. Universidade Federal do Pará.

Bastaits, K., \& Mortelmans, D. (2016). Parenting as Mediator Between Post-divorce Family Structure and Children's Well-being. Journal of Child and Family Studies, 25(7), 2178-2188. doi: 10.1007/s10826-016-0395-8

Bastaits, K., Ponnet, K., \& Mortelmans, D. (2012). Parenting of divorced fathers and the association with children's self-esteem. J Youth Adolesc, 41(12), 1643-1656. doi: 10.1007/s10964-012-9783-6

Böing, E., Crepaldi, M. A., \& Moré, C. L. O. (2008). Pesquisa com famílias: aspectos teórico-metodológicos. Paidéia, 18(40), 251-266.

Bossardi, C. N. (2011). Relação do engajamento parental e relacionamento conjugal no investimento com os filhos. Dissertação (Mestrado em Psicologia). Programa de Pós-Graduação em Psicologia. Universidade Federal de Santa Catarina, Brasil.

Bossardi, C. N., Gomes, L. B., Vieira, M. L., \& Crepaldi, M. A. (2013). Engajamento paterno no cuidado a crianças de 4 a 6 anos. Psicologia Argumento, Curitiba.

Brito, L. M. T. (2007). Família Pós-Divórcio: A Visão dos Filhos. Psicologia Ciência E Profissão, 27(1), 32-45.

Bueno, R. K. (2014). Relações entre envolvimento paterno com filhos adotivos e estrutura familiar. Dissertação (Mestrado em Psicologia). Programa de Pós-Graduação em Psicologia. Universidade Federal de Santa Catarina, Brasil.

Cabrera, N. J., Tamis-LeMonda, C. S., Bradley, R. H., Hofferth, S., \& Lamb, M. E. (2000). Fatherhood in the twenty-first century. Child Development, 71(1), 127-36.doi: $10.1111 / 1467-8624.00126$

Actualidades en Psicología, 32(124), 2018, 91-109 
Cezar-Ferreira, V. A. da M., \& Macedo, R. M. S. (2016). Guarda Compartilhada: Uma visão psicojurídica (Artmed). Porto Alegre: Artmed.

Cia, F., Williams, L. C. deA., \& Aiello, A. L. R. (2005). As influências paternas no desenvolvimento infantil: revisão da literatura. Psicologia Escolar E Educacional, 9(2), 225-233.

Cheadle, J. E., Amato, P. R., \& King, V. (2010). Patterns of nonresident father contact. Demography, 47(1), 205-225. doi: 10.1353/dem.0.0084

Cohen, O., Finzi-Dottan, R., \& Tangir-Dotan, G. (2014). The Fatherhood Experience of Divorced Custodial Fathers in Israel. Family Relations, 63(5), 639-653. doi: 10.1111/Fare.12092

Cookston, J. T., Braver, S. L., Griffin, W. A., De Luse, S. R., \& Miles, J. C. (2007). Effects of the Dads for Life intervention on interparental conflict and coparenting in the two years after divorce. Fam Process, 46(1), 123-137. Recuperado de http:// www.ncbi.nlm.nih.gov/pubmed/17375733

Creswell, J. W. (2007). Projeto de pesquisa: métodos qualitativo, quantitativo e misto (3rd ed.). Porto Alegre: Artmed.

Degarmo, D. S., \& Forgatch, M. S. (2012). A confidant support and problem solving model of divorced fathers' parenting. Am J Community Psychol, 49(1-2), 258-269. doi: 10.1007/s10464-011-9437-y.

Dubeau, D., Devault, A., \& Paquette, D. (2009). L'engagement des parents: un concept à multiples facettes. In D. Dubeau, A. Devault, \& G. Forget (Eds.), La paternité au XXI sièle. Québec, Canada: Les Presses de l'Université Laval.

Elam, K. K., Sandler, I., Wolchik, S., \& Tein, J.-Y. (2016). Non-residential father-child involvement, interparental conflict and mental health of children following divorce: A person-focused approach. Journal of Youth and Adolescence, 45(3), 581 593. doi: 10.1007/s10964-015-0399-5

Fabricius, W. V, \& Luecken, L. J. (2007). Postdivorce living arrangements, parent conflict, and long-term physical health correlates for children of divorce. Journal of Family Psychology, 21(2), 195-205. doi: 10.1037/0893-3200.21.2.195

Finley, G. E., \& Schwartz, S. J. (2006). Parsons and Bales revisited: Young adult children's characterization of the fathering role. Psychology of Men \& Masculinity, 7(1), 42-55. doi: 10.1037/1524-9220.7.1.42

Flouri, E. (2006). Non-resident fathers' relationships with their secondary school age children: determinants and children's mental health outcomes. J Adolesc, 29(4), 525-538. doi: 10.1016/j.adolescence.2005.08.004

Flouri, E., Narayanan, M. K., \& Midouhas, E. (2015). The cross-lagged relationship between father absence and child problem behaviour in the early years. Child: Care, Health and Development, 41(6), 1090-097. doi: 10.1111/cch.12236

Gomes, L. B., Bossardi, C. N., Cruz, R. M., Crepaldi, M. A., \& Vieira, M. L. (2014). Propriedades psicométricas de instrumentos de avaliação do envolvimento paterno: revisão de literatura. Avaliação Psicológica, 13(47), 19-27. 
Greene, S. M., Anderson, E. R., Forgatch, M. S., DeGarmo, D. S., \& Hetherington, E. M. (2016). Risco e resiliência após o divórcio. In Processos Normativos da Família (Artmed, pp. 102-127). Porto Alegre: Artmed.

Grzybowski, L. S. (2007). Parentalidade em tempo de mudanças: desvelando o envolvimento parental após o fim do casamento. Tese (doutorado). Faculdade de Psicologia. Programa de Pós-Graduação em Psicologia. PUCRS.

Grzybowski, L. S., \& Wagner, A. (2010a). Casa do pai, casa da mãe: a coparentalidade após o divórcio. Psicologia: Teoria E Pesquisa, 26(1), 77-87. doi: 10.1590/S010237722010000100010

Grzybowski, L. S., \& Wagner, A. (2010b). O Envolvimento Parental Após a Separação. Psicologia-Reflexao E Critica, 23(2), 289-298.

Hack, S. M. P. K., \& Ramires, V. R. R. (2010). Adolescência e divórcio parental: continuidades e rupturas dos relacionamentos. Psicologia Clínica, 22(1), 85-97. doi: 10.1590/S0103-56652010000100006

Halme, N., Åstedt-Kurki, P., \& Tarkka, M.-T. (2009). Fathers' Involvement with their Preschool-age Children: How Fathers Spend Time with Their Children in Different Family Structures. Child \& Youth Care Forum, 38(3), 103-119. doi: 10.1007/s10566-009-9069-7

Hendricks, C. S., Cesario, S. K., Murdaugh, C., Gibbons, M. E., Servonsky, E. J., Bobadilla, R. V., Tavakoli, A. (2005). The influence of father absence on the self-esteem and self-reported sexual activity of rural southern adolescents. ABNF J, 16(6), 124-131. Recuperado de http://www.ncbi.nlm.nih.gov/pubmed/16382796

Hetherington, E. M., \& Kelly, J. (2002). For better or for worse: divorce recon- sidered (Norton \& C). New York.

IBGE. (2014). Instituto Brasileiro de Geografia e Estatistica. Retrieved from http://www. brasil.gov.br/cidadania-e-justica/2015/11/em-10-anos-taxa-de-divorcioscresce-mais-de-160-no-pais

Kalil, A., Mogstad, M., Rege, M., \& Votruba, M. (2011). Divorced fathers' proximity and children's long-run outcomes: evidence from Norwegian registry data. Demography, 48(3), 1005-1027. doi: 10.1007/s13524-011-0046-z

Kalmijn, M. (2015). Father-Child Relations after Divorce in Four European Countries: Patterns and Determinants. Comparative Population Studies, 40, 251-276. doi: 10.12765/CPoS-2015-10en

King, V., \& Sobolewski, J. M. (2006). Nonresident fathers' contributions to adolescent well-being. Journal of Marriage and Family, 68(3), 537-557. doi: 10.1111/J.17413737.2006.00274.X

Klaus, D., Nauck, B., \& Steinbach, A. (2012). Relationships to stepfathers and biological fathers in adulthood: Complementary, substitutional, or neglected? Advances in Life Course Research, 17(3), 156-167. doi: 10.1016/J.Alcr.2012.01.006 
Lamb, M. E. (1992). O Papel do Pai em Mudança. Análise Psicológica, 1, 19-34.

Lamb, M. E. (1997). Fathers and child development: an introductory overview and guide. In The role of the father in child development (3rd ed., pp. 1-18). New York: John Wiley \& Sons, Inc.

Lamela, D., Castro, M., \& Figueiredo, B. (2010). Pais por inteiro: avaliação preliminar da eficácia de uma intervenção em grupo para pais divorciados. Psicologia: Reflexão E Crítica, 23(2), 334-344. doi: 10.1590/S0102-79722010000200016

Lamela, D., \& Figueiredo, B. (2016). Co-parenting after marital dissolution and children's mental health: a systematic review. Jornal de Pediatria, 92(4), 331-342. doi: 10.1016/j.jped.2015.09.011

Lamela, D., Nunes-Costa, R., \& Figueiredo, B. (2010). Modelos teóricos das relações coparentais: revisão crítica. Psicologia Em Estudo, 15(1), 205-216. doi: 10.1590/ S1413-73722010000100022

Lewis, C., \& Dessen, M. A. (1999). O Pai no Contexto Familiar. Psicologia: Teoria e Pesquisa. Recuperado de https://revistaptp.unb.br/index.php/ptp/article/view/1485

Maturana, H. (2014a). A Ontologia da Realidade. (C. Magro, M. Graciano, \& N. Vaz, Eds.) (2nd ed.). Belo Horizonte: Humanitas.

Maturana, H. (2014b). Cognição, Ciência e Vida Cotidiana. (C. Magro \& V. Paredes, Eds.) (2nd ed.). Belo Horizonte: EditoraUFMG.

Maturana, H. R., \& Varela, F. J. (2001). A Árvore do Conhecimento. As bases biológicas da compreensão humana (9th ed.). São Paulo: Palas Athena.

Maturana, H., \& Yáñez, X. D. (2015). El arvol del vivir. Santiago, Chile: MVP Editores.

McGoldrick, M., \& Shibusawa, T. (2016). O ciclo vital familiar. In Processos Normativos da Familia (Artmed, pp. 375-398). Porto Alegre: Artmed.

Mendle, J., Harden, K. P., Turkheimer, E., Hulle, C. A. Van, Brian, M., Onofrio, D., Emery, R. E. (2009). Associations Between Father Absence and Age of First Sexual Intercourse. Child Dev., 80(5), 1463-1480. doi: 10.1111/j.14678624.2009.01345.x.Associations

Modecki, K. L., Hagan, M., Sandler, I., \& Wolchik, S. (2015). Latent profiles of non-residential father engagement six years after divorce predict long term offspring outcomes. J Clin Child Adolesc Psychol., 44(1), 123-136. doi: 10.1080/15374416.2013.865193.Latent

Nobles, J. (2011). Parenting From Abroad: Migration, Nonresident Father Involvement, and Children's Education in Mexico. Journal of Marriage and Family, 73(4), 729746. doi: 10.1111/J.1741-3737.2011.00842.X

Owen, J., \& Rhoades, G. K. (2012). Reducing Interparental Conflict Among Parents in Contentious Child Custody Disputes: An Initial Investigation of the Working Together Program. Journal of Marital and Family Therapy, 38(3), 542-555. 
Scott, M. E., Booth, A., King, V., \& Johnson, D. R. (2007). Postdivorce father-adolescent closeness. Journal of Marriage and Family, 69(5), 1194-1209. doi: 10.1111/J.17413737.2007.00441.X

Silva, L. (2012). Boas práticas dos programas psicoeducacionais para pais separados/divorciados. Tese de mestrado, Psicologia (Secção de Psicologia Clínica e da Saúde - Núcleo de Psicoterapia Cognitiva-Comportamental e Integrativa), Universidade de Lisboa, Faculdade de Psicologia, 2012. Recuperado de http://repositorio.ul.pt/// handle/10451/8287

Silva, M. da R. (2003). Sentimentos sobre a paternidade e envolvimento de pais que residem e pais que não-residem com seus filhos. Dissertação (Mestrado). Curso de Pós-Graduação em Psicologia do Desenvolvimento. Universidade Federal do Rio Grande do Sul.

Stamps, K., Booth, A., \& King, V. (2009). Adolescents With Nonresident Fathers: Are Daughters More Disadvantaged Than Sons? Journal of Marriage and Family, 71(3), 650-662.

Vasconcellos, M. J. E. de. (2010). Pensamento Sistêmico. O novo paradigma da ciência (9th ed.). Campinas, SP: Papirus.

Vieira, M. L., Bossardi, C. N., Gomes, L. B., Bolze, S. D. A., Crepaldi, M. A., \& Piccinini, C. A. (2014). Paternidade no Brasil: revisão sistemática de artigos empíricos. Arquivos Brasileiros de Psicologia, 66(2), 36-52.

Warshak, R. A. (2014). Social science and parenting plans for young children: A consensus report. Psychology, Public Policy, and Law, 20(1), 46-67. doi: 10.1037/law0000005

Yu, T. Y., Pettit, G. S., Lansford, J. E., Dodge, K. A., \& Bates, J. E. (2010). The Interactive Effects of Marital Conflict and Divorce on Parent - Adult Children's Relationships. Journal of Marriage and Family, 72(2), 282-292. doi: 10.1111/J.17413737.2010.00699.X

Zordan, E. P., Wagner, A., \& Mosmann, C. (2012). O perfil de casais que vivenciam divórcios consensuais e litigiosos : uma análise das demandas judiciais. PsicoUSF, 17(2), 185-194.

Recibido: 22 de mayo del 2017

Aceptado: 21 de marzo del 2018 